\title{
Changing paradigm in pancreatic cancer: from adjuvant to neoadjuvant chemoradiation
}

\author{
Justin D. Anderson ${ }^{1}$, Wen Wan $^{2}$, Brian J. Kaplan ${ }^{3}, J^{\prime}$ nnifer Myers ${ }^{4}$, Emma C. Fields $^{1}$ \\ ${ }^{1}$ Department of Radiation Oncology, Massey Cancer Center, ${ }^{2}$ Department of Biostatistics, ${ }^{3}$ Department of Surgical Oncology, ${ }^{4}$ Department of \\ Hematology Oncology, Virginia Commonwealth University, Richmond, VA 23298, USA \\ Contributions: (I) Conception and design: JW Anderson, EC Fields; (II) Administrative support: JW Anderson; (III) Provision of study materials or \\ patients: BJ Kaplan, J Myers, EC Fields; (IV) Collection and assembly of data: JW Anderson; (V) Data analysis and interpretation: W Wan; (VI) \\ Manuscript writing: All authors; (VII) Final approval of manuscript: All authors. \\ Correspondence to: Emma C. Fields, MD. Department of Radiation Oncology, Virginia Commonwealth University, Richmond, VA 23298-0058, USA. \\ Email: emma.fields@vcuhealth.org.
}

Background: Historically, management of pancreatic cancer has been determined based on whether the tumor was amenable to resection and all patients deemed resectable received curative intent surgery followed by adjuvant therapy with chemotherapy $(\mathrm{CT}) \pm \mathrm{RT}$. However, patients who undergo resection with microscopic (R1) positive margins have inferior rates of survival. The purpose of this study is to identify patients who have undergone pancreatectomy for pancreatic cancer, determine the surgical margins, types of adjuvant therapies given and patterns of failure. Our hypothesis was that in patients who have surgery without pre-operative therapy, there is a high rate of $\mathrm{R} 1$ resections and subsequent local recurrence, despite adjuvant therapy.

Methods: Seventy-one patients with curative resections for pancreatic cancer between 2003 and 2015 were reviewed. Tumor stage, margin status, distance to closest margin, receipt of adjuvant therapy and length of survival were collected. Patients were divided into two groups based on whether they received adjuvant CT + RT ( $\mathrm{n}=37)$ or CT alone (n=37). Patients were further divided based on whether resection was R1 (n=29) or R0 ( $\mathrm{n}=42)$. Wilcoxon survival tests and Cox proportional hazards regression models were performed to determine the effects of CT + RT vs. CT alone, stratified by surgical margin status.

Results: Of the 29 patients (39\%) who had R1, 15 received CT + RT and 14 received only CT. Patients who received CT + RT experienced a significantly longer period of PFS (13 vs. 7.5 mos, $\mathrm{P}=0.03)$ than patients who received CT alone. However, there was no significant difference found in time to death post cancer resection between $\mathrm{CT}+\mathrm{RT}$ vs. CT alone $(\mathrm{P}=0.73)$. Of the 42 patients with $\mathrm{R} 0,21$ received $\mathrm{CT}+\mathrm{RT}$ and 21 received CT. There was a trend towards increase in PFS in patients treated with CT + RT (25 vs. 17 months, $\mathrm{P}=0.05)$, but there was no significant increase in time to death compared to patients treated with CT alone $(\mathrm{P}=0.53$. Of the 36 patients with CT + RT, 21 had R0 and 15 had R1. Patients with R0 were more likely to have longer PFS ( 25 vs. 13 months, $\mathrm{P}=0.06$ ), but there was no significant difference in time to death compared to patients with CT alone $(\mathrm{P}=0.68)$.

Conclusions: After curative resection, the addition of RT to CT improves PFS in both R0 and R1 settings. However, patients with R1 have significantly worse PFS and OS compared to patients with R0 and even aggressive adjuvant therapy does not make up for the difference. The paradigm has shifted and now for patients with resectable pancreatic cancers we recommend neoadjuvant CT + RT to improve RT targeting and treatment response assessment and most importantly, improve chances of obtaining R0.

Keywords: Pancreatic cancer; adjuvant therapy; chemotherapy (CT); radiotherapy

Submitted Jul 14, 2016. Accepted for publication Sep 02, 2016.

doi: 10.21037/jgo.2016.11.09

View this article at: http://dx.doi.org/10.21037/jgo.2016.11.09 


\section{Introduction}

In 2016 an estimated 53,070 people will be diagnosed with pancreatic cancer and of those 41,780 will succumb to the disease (1). Traditionally, the management of pancreatic cancer has been determined based on whether the tumor was amenable to resection, was unresectable, or metastatic at presentation. To determine resectability of a tumor at the time of presentation is difficult, but is typically based on either computed tomography or magnetic resonance imaging of the tumor and its relationship to surrounding blood vessels. There are various definitions of surgical resectabilty from the Americas Hepato-PancreatoBiliary Association (AHPBA), MD Anderson, National Comprehensive Cancer Network (NCCN) and Alliance, but the mainstay of resectability is a tumor that is free from contact of the major arterial and venous structures (2).

Resection status is an important prognostic factor for outcome and survival of pancreatic cancer $(3,4)$. However, at the time of diagnosis, many patients have borderline or unresectable cancer, involving the vasculature. Even if a patient's cancer is deemed "resectable" it is possible for the patient to have positive microscopic margins on final pathology. Most commonly, the retroperitoneal margin or circumferential margin is positive, due to the technically difficult location of the tumor (5). Patients with positive microscopic margins (R1) have a poorer prognosis compared to patients with negative margins (R0) (20.3 months for R0 vs. 10.3 months for R1) (6).

In the United States, radiation therapy has customarily been used in the adjuvant setting for resected pancreatic cancer $(7,8)$. However, internationally the role of adjuvant radiation is controversial as there are European studies which show no benefit to adjuvant treatment and in fact show a detriment to its use (9). Due to different patient inclusion criteria, radiation doses and schedules, these studies are difficult to compare head to head. One striking difference is that the largest study claiming a detriment to the use of radiotherapy included patients with positive margins (9).

The purpose of this study is to determine whether adjuvant radiotherapy and/or chemotherapy (CT) can compensate for microscopic positive margins at the time of resection. Our hypothesis is that in patients who have surgery without pre-operative therapy, there is a high rate of $\mathrm{R} 1$ resections and subsequent local recurrence, despite adjuvant therapy.

\section{Methods}

\section{Patient Information}

Under Virginia Commonwealth University (VCU) institutional review board approval, a retrospective analysis of patients treated at VCU Health for pancreatic cancer was performed to determine patient criteria and outcomes. Records for 71 patients treated with curative intent for pancreatic cancer at VCU were available in the electronic medical records from 2003 to 2015.

Tumor stage, margin status (positive, negative), distance to closest margin, receipt of adjuvant therapy and length of survival were collected and evaluated. Patients were divided into two groups based on whether they received adjuvant CT plus radiation therapy $(n=36)$ or CT alone $(n=35)$. Patients were further divided based on whether resection was R1 (n=29) or R0 (n=42) (Figure 1).

\section{Treatment details}

$\mathrm{CT}$ and radiation given throughout the study was consistent with few exceptions. Most patients $(85.9 \%, \mathrm{n}=61)$ received gemcitabine at standard doses given over 28 day cycles for a median of 4 cycles (range, 1-6 cycles). Other less common regimens included: gemcitabine plus cisplatin and taxotere $(n=1)$, gemcitabine and abraxane $(n=1)$, folfirnox $(n=3)$, streptozocin and Adriamycin $(n=1)$, capecitabine $(n=1)$, cisplatin and etoposide $(\mathrm{n}=2)$, and 5 -fluorouracil $(\mathrm{n}=1)$. External beam radiation therapy was given with a median dose of 50.4 Gy in 1.8 Gy fractions (range, 23.4-50.4 Gy). About half of the patients were treated with $3 \mathrm{D}$ conformal technique $(46.5 \%, \mathrm{n}=33)$ and the rest with intensity modulated radiation therapy (IMRT). Patients received either 5-fluoruracil or capecitabine as concurrent CT during radiation therapy.

\section{Statistical analysis}

Baseline characteristics including $\mathrm{T}$ and $\mathrm{N}$ stages were summarized by frequency and proportion per group. Patients in T stage were dichotomized into two groups: low (T 1-2) and high (T 3-4) T stages. Kaplan-Meier curves and Wilcoxon tests were used to illustrate the difference in the cumulative incidence of time to recurrence and/or death between the patients who did and did not receive radiation therapy or CT, with or without stratification of positive/ 


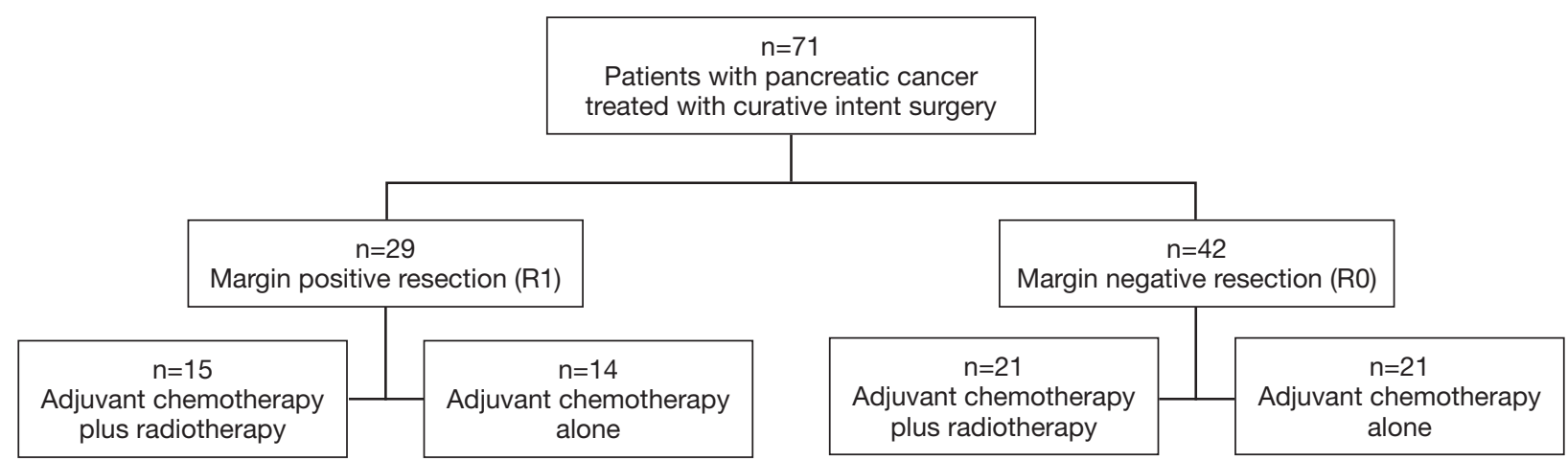

Figure 1 Treatment schema showing all patients divided based on margin status and then receipt of CT vs. chemoradiation. CT, chemotherapy.

negative surgical margin, respectively. Cox proportional hazards regression models were performed to model time to recurrence/death to determine the effects of radiation and CT therapy versus CT alone adjusted by potential risk factors such as $\mathrm{T}$ and $\mathrm{N}$ stages, surgical margin, and/or interaction effect between recurrence and $\mathrm{T}$ stage. All computations were performed using SAS ${ }^{\circledR}$ 9.4 Software.

\section{Results}

The median age of patients at the time of pancreatic surgery was 62 years old. Approximately half the patients were male $(47.9 \%)$ and half female $(52.1 \%) .71 .8 \%(\mathrm{n}=51)$ of cancers were located in the head of the pancreas, $8.4 \%(n=6)$ were located in the tail of the pancreas, $7.0 \%(\mathrm{n}=5)$ were in the body, and $4.2 \%(\mathrm{n}=5)$ in the neck/uncinate process, and 5.6\% $(n=4)$ were unreported. The majority $(69.0 \%, n=49)$ of the cancers were moderately differentiated at final pathology, $8.5 \%, \mathrm{n}=6$ patients had well differentiated adenocarcinoma, $14.1 \%, \mathrm{n}=10$ had poorly differentiated adenocarcinoma and $8 \% \mathrm{n}=6$ were not reported. The median follow up of all patients was 26.6 months. The patient characteristics are outlined in Table 1.

\section{Margin positive resection and outcomes}

Of the 29 patients (40.8\%) who had R1 margins, 15 received CT plus radiation therapy and 14 received CT only. Patients who received CT plus radiation therapy experienced a significantly longer period of progression free survival (13 vs. 7.5 months, $\mathrm{P}=0.03$ ) than patients who received CT alone (Figure $2 A$ ). However, there was no significant difference found in time to death post cancer resection between $\mathrm{CT}$ plus radiation therapy vs. CT alone $(\mathrm{P}=0.73$ ) (Figure 2B).

\section{Margin negative resection and outcomes}

Of the 42 patients (59.2\%) who had R0 margins, 21 received CT plus radiation therapy and 21 received CT alone. There was a trend towards an increase in progression free survival in patients treated with CT plus radiation therapy (25 vs. 17 months, $\mathrm{P}=0.05$ ), but there was no significant increase in time to death compared to patients treated with CT alone $(\mathrm{P}=0.53)$ (Figures 3).

\section{Radiation therapy and outcomes}

Of the 36 patients with CT plus radiation therapy, 21 had R0 and 15 had R1. Patients with R0 were more likely to have longer progression free survival ( 25 vs. 13 months, $\mathrm{P}=0.06$ ), but there was no significant difference in time to death compared to patients with CT alone $(\mathrm{P}=0.68)$ (Figure 4).

\section{All patient outcomes}

Overall, patients with R0 margins were more likely than patients with $\mathrm{R} 1$ margins to have longer progression free survival (median 21 vs. 11 months, $\mathrm{P}=0.03$ ). We did not detect a difference in overall survival between patients with $\mathrm{R} 0$ vs. $\mathrm{R} 1$ margins $(\mathrm{P}=0.52)$ (Figure 5).

\section{Discussion}

This study adds to the literature by showing the importance of $\mathrm{R} 0$ resection in patients with pancreatic cancer and 
Table 1 Patient characteristics

\begin{tabular}{|c|c|c|c|}
\hline Characteristics & $\begin{array}{c}\text { All patients, } \\
n=71(\%)\end{array}$ & $\begin{array}{l}\text { Ro group, } \\
n=42(\%)\end{array}$ & $\begin{array}{l}\text { R1 group, } \\
n=29(\%)\end{array}$ \\
\hline \multicolumn{4}{|l|}{ Gender } \\
\hline Male & $34(47.9)$ & $21(50.0)$ & $13(44.8)$ \\
\hline Female & $37(52.1)$ & $21(50.0)$ & $16(55.2)$ \\
\hline \multicolumn{4}{|l|}{ Age (years) } \\
\hline Median & 62.0 & 63.4 & 61.3 \\
\hline Range & $23.6-84.2$ & $23.6-84.2$ & $42.0-80.9$ \\
\hline \multicolumn{4}{|l|}{ Closest margin $(\mathrm{cm})$} \\
\hline Median & 0.1 & 0.1 & 0 \\
\hline Range & $<0.01-1.1$ & $<0.01-1.1$ & NA \\
\hline Positive & 29 & 0 & 29 \\
\hline Not recorded & 19 & 19 & 0 \\
\hline \multicolumn{4}{|l|}{ T stage } \\
\hline T 1-2 & $11(15.5)$ & $9(21.4)$ & $3(10.3)$ \\
\hline T 3-4 & 55 (77.5) & $31(73.8)$ & 23 (79.3) \\
\hline Not recorded & $5(7.0)$ & $2(4.8)$ & $3(10.3)$ \\
\hline \multicolumn{4}{|l|}{$\mathrm{N}$ stage } \\
\hline LN positive (N1) & $42(59.2)$ & $24(57.2)$ & $18(62.1)$ \\
\hline LN negative (NO) & $27(38.0)$ & $16(38.1)$ & $11(37.9)$ \\
\hline Not recorded & $2(2.8)$ & $2(4.8)$ & 0 \\
\hline \multicolumn{4}{|c|}{ Tumor differentiation } \\
\hline Low & $6(8.5)$ & $4(9.5)$ & $2(6.9)$ \\
\hline Moderately & $49(69.0)$ & $25(59.5)$ & $24(82.8)$ \\
\hline Poorly & $10(14.1)$ & $9(21.4)$ & $1(3.4)$ \\
\hline Not recorded & $6(8.5)$ & $4(9.5)$ & $2(6.9)$ \\
\hline \multicolumn{4}{|l|}{ Tumor location } \\
\hline Head & $51(71.8)$ & $31(73.8)$ & $20(69.0)$ \\
\hline Neck/Uncinate & $5(7.0)$ & $2(4.8)$ & $3(10.3)$ \\
\hline Body & $5(7.0)$ & $4(9.5)$ & $1(3.4)$ \\
\hline Tail & $6(8.5)$ & $3(7.1)$ & $3(10.3)$ \\
\hline Not recorded & $4(5.6)$ & $2(4.8)$ & $2(6.9)$ \\
\hline \multicolumn{4}{|l|}{ Radiation therapy } \\
\hline Treated & $36(50.1)$ & $21(50.0)$ & 15 (51.7) \\
\hline \multicolumn{4}{|l|}{ CT } \\
\hline Treated & $71(100.0)$ & $42(100.0)$ & $29(100.0)$ \\
\hline
\end{tabular}

CT, chemotherapy. demonstrates that the rate of $\mathrm{R} 1$ resection remains high even in carefully selected patients $(6,10,11)$. Nearly $40 \%$ of patients considered resectable in our study had positive margins, which is consistent with the current literature $(6,12)$. After curative resection, the addition of radiation therapy to CT improved progression free survival in both R0 and R1 settings. However, patients with R1 had significantly worse progression free survival compared to patients with R0 and even aggressive adjuvant therapy did not make up for the difference.

In other gastrointestinal malignancies such as esophageal and rectal cancers, radiation therapy is used preoperatively and has shown to improve treatment compliance, increase rates of curative surgery with down-staging, have better tumor oxygenation and decreased toxicity compared with postoperative therapy (13-15). In pancreatic cancer, the standard therapy for decades has been to resect upfront if possible and give $\mathrm{CT}$ and radiation therapy postoperatively. However, administering radiation postoperatively to the pancreatic bed comes with similar challenges as with other gastrointestinal sites. Treatment with adjuvant therapy is frequently delayed due to surgery and recovery time which potentially gives any residual cancer extra time to progress. Lack of receipt of some or all adjuvant treatment is also common due to decreased nutrition and overall wellness. In fact, anywhere from $25-50 \%$ of patients do not complete adjuvant therapy after pancreatic tumor resection for various reasons (16). Other challenges of using adjuvant therapy include determining the areas at highest risk of recurrence postoperatively and administering a high enough dose of radiation in the setting of $\mathrm{R} 1$ margins without giving a toxic dose to surrounding organs, particularly the bowel and kidneys.

A recent study from The University of Texas MD Anderson Cancer Center reported higher rates of completion of multimodality therapy in the neoadjuvant compared to the adjuvant setting; further suggesting patients are more likely to receive the full benefit of all modalities with neoadjuvant treatment of CT with or without radiation (17). One of the primary benefits of preoperative treatment is to "test the biology" of the cancer and not put patients through the operation who have micrometastatic or rapid progressive disease at the time of diagnosis (18). Neoadjuvant therapy will delay surgery long enough that patients unlikely to benefit from the procedure will not be subject to unwanted consequences. This could save the patient from unnecessary suffering and allow for palliative care to be administered earlier. 

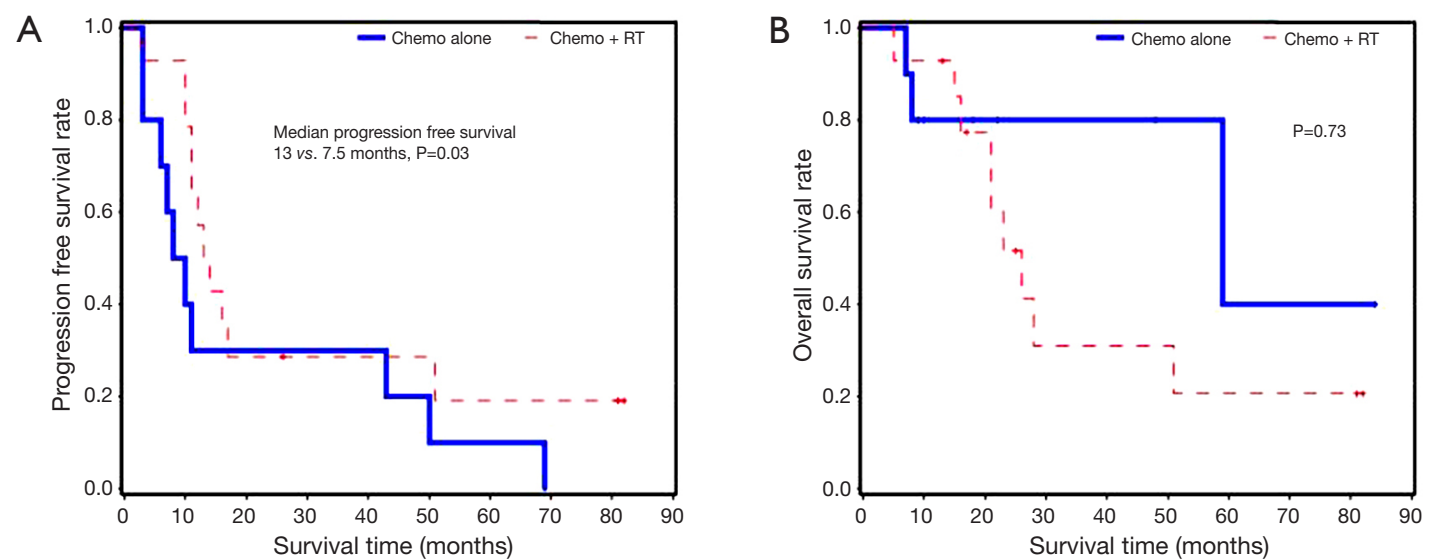

Figure 2 Kaplan-Meier curves for patients with margin positive resection. (A) Progression free survival of patients receiving CT vs. chemoradiation; (B) overall survival of patients receiving CT vs. chemoradiation. CT, chemotherapy.
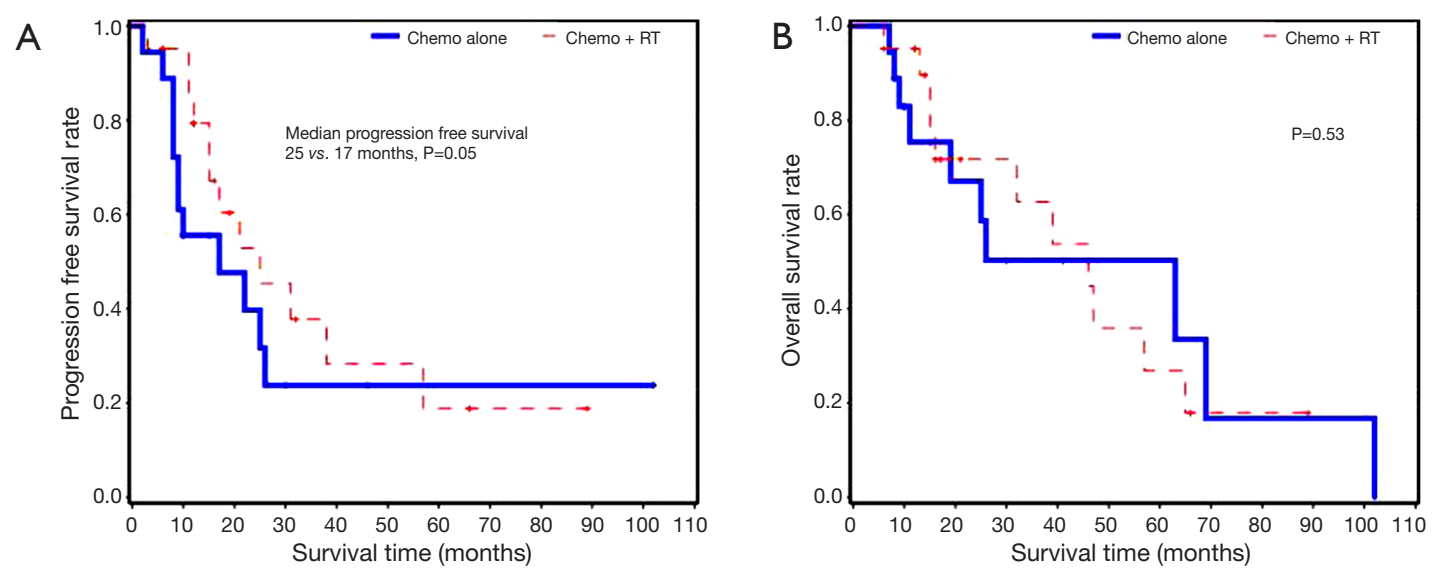

Figure 3 Kaplan-Meier curves for patients with margin negative resection. (A) Progression free survival of patients receiving CT vs. chemoradiation; (B) overall survival of patients receiving CT vs. chemoradiation. CT, chemotherapy.
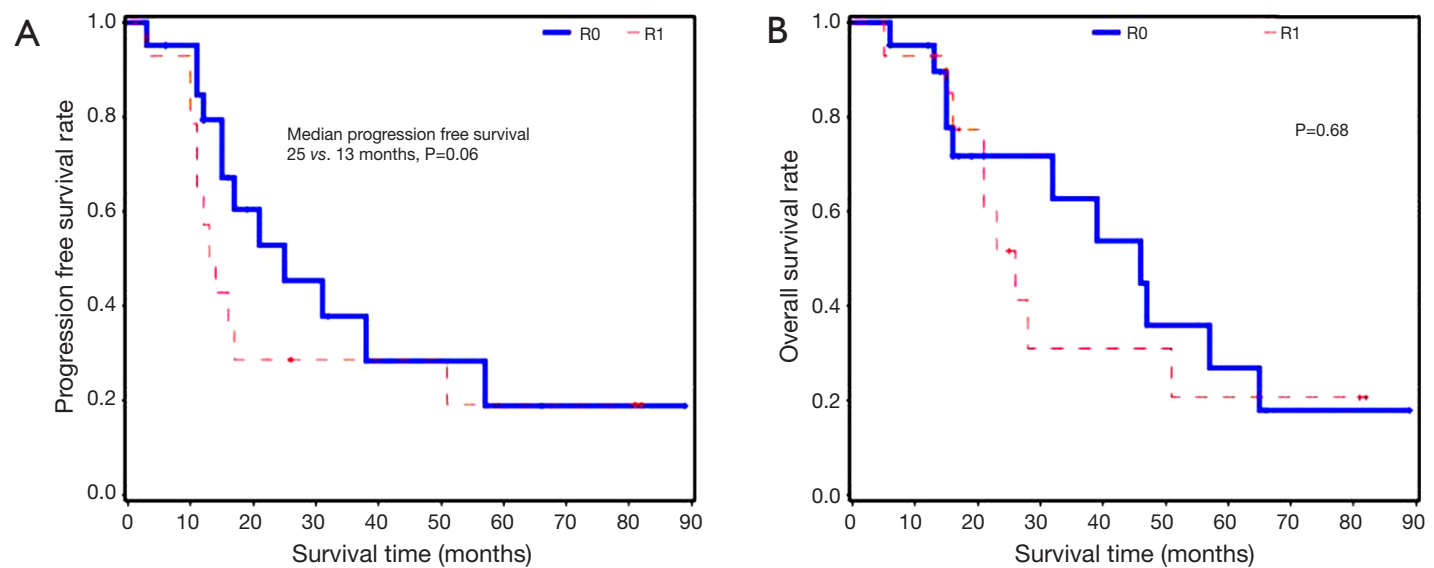

Figure 4 Kaplan-Meier curves for patients who received chemoradiation. (A) Progression free survival of patients with R0 vs. R1 resection; (B) overall survival of patients with R0 vs. R1 resection. 

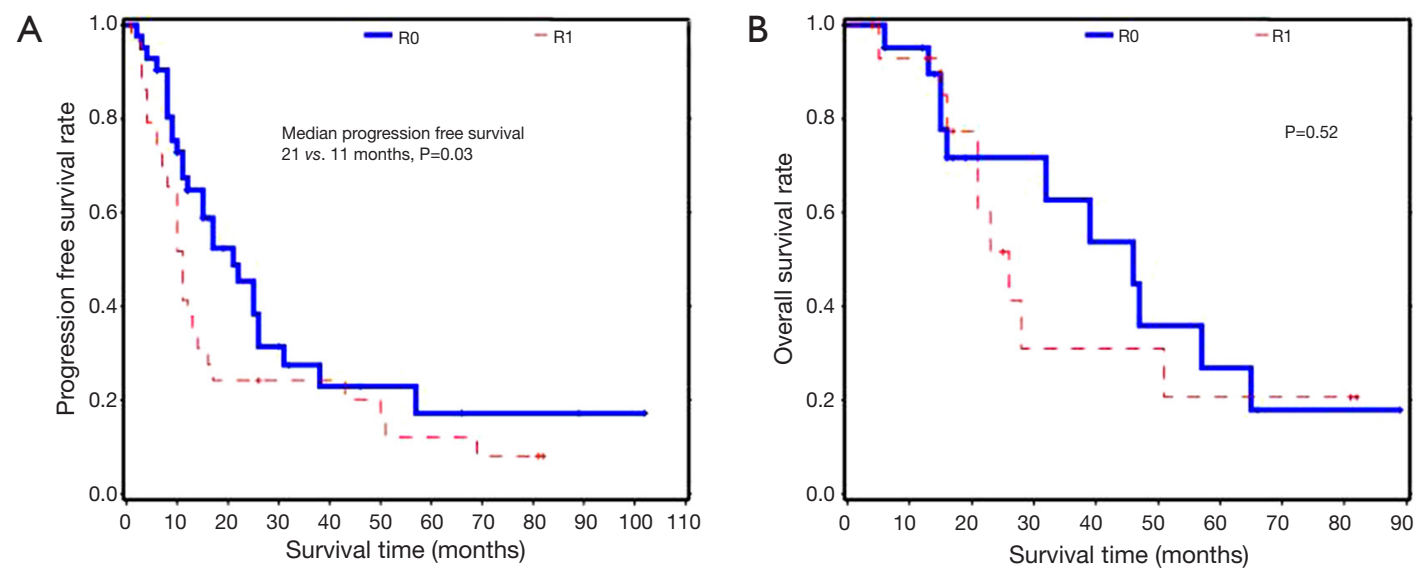

Figure 5 Kaplan-Meier curves for all patients. (A) Progression free survival of patients with R0 vs. R1 resection; (B) overall survival of patients with R0 vs. R1 resection.

For patients with a good response to neoadjuvant therapy, there are lower risks of $\mathrm{R} 1$ resection and their postoperative CT may be tailored to their drug susceptibility (19-21). Based on our results, it is reasonable to propose that neoadjuvant therapy be considered for all nonmetastatic pancreatic cancers to increase the chances of R0 margin status. Even when patients are considered resectable they have a high risk of cancer positive margins (6). It is common for patients with borderline resectability to receive neoadjuvant therapy to help them become candidates for surgery. The literature shows that (41-47\%) patients with borderline resectability can down-stage and become resectable with neoadjuvant therapy $(19,20)$.

Concerns have been raised about delaying surgery because of the risk of the cancer progressing to unresectable status before the patient receives surgery (3). Despite this risk, the benefits of neoadjuvant therapy are hard to overlook, including increased rates of R0 resections, improved tolerance of treatment, better effect of treatment due to decreased hypoxia, and less patients receiving a morbid operation in the setting of micrometastatic disease. Using neoadjuvant therapy even for patients considered resectable is not a novel idea and is deserving of increased consideration from the medical community $(22,23)$.

Our study has potential bias because of the lack of randomization and its retrospective nature. The power of the study is also limited due to the small sample size. Despite the limitations to our study, our results are similar to many other larger studies and show an increasing need to further investigate the use of neoadjuvant therapy for all patients with non-metastatic pancreatic cancer.

\section{Conclusions}

The traditional treatment paradigm of surgical resection followed by adjuvant chemoradiation leaves a high rate of $\mathrm{R} 1$ resections and adjuvant therapy is unable to compensate for residual tumor, with increased rates of progression and detriments to OS. This study adds to the growing literature in favor of pre-operative chemoradiation for all potentially resectable pancreatic adenocarcinomas in order to reduce the risk of having $\mathrm{R} 1$ positive margins and increase progression free survival and overall survival.

\section{Acknowledgements}

None.

\section{Footnote}

Conflicts of Interest: The authors have no conflicts of interest to declare.

Ethical Statement: The study was approved by institutional ethics board of Virginia Commonwealth University (VCU) (No. HM20004325) and written informed consent was obtained from all patients.

\section{References}

1. Howlader N, Noone AM, Krapcho M, et al. SEER Cancer Statistics Review, 1975-2013, National Cancer Institute. Bethesda, MD. Available online: http://seer.cancer.gov/ 
csr/1975_2013/

2. Ryan DP, Hong TS, Bardeesy N. Pancreatic adenocarcinoma. N Engl J Med 2014;371:1039-49.

3. Vincent A, Herman J, Schulick R, et al. Pancreatic cancer. Lancet 2011;378:607-20.

4. Cooper AB, Tzeng CW, Katz MH. Treatment of borderline resectable pancreatic cancer. Curr Treat Options Oncol 2013;14:293-310.

5. Verbeke CS, Menon KV. Redefining resection margin status in pancreatic cancer. HPB (Oxford) 2009;11:282-9.

6. Neuzillet C, Sauvanet A, Hammel P. Prognostic factors for resectable pancreatic adenocarcinoma. J Visc Surg 2011;148:e232-43.

7. Further evidence of effective adjuvant combined radiation and chemotherapy following curative resection of pancreatic cancer. Gastrointestinal Tumor Study Group. Cancer 1987;59:2006-10.

8. Regine WF, Winter KA, Abrams R, et al. Fluorouracilbased chemoradiation with either gemcitabine or fluorouracil chemotherapy after resection of pancreatic adenocarcinoma: 5-year analysis of the U.S. Intergroup/ RTOG 9704 phase III trial. Ann Surg Oncol 2011;18:1319-26.

9. Neoptolemos JP, Stocken DD, Friess H, et al. A randomized trial of chemoradiotherapy and chemotherapy after resection of pancreatic cancer. N Engl J Med 2004;350:1200-10.

10. Campbell F, Smith RA, Whelan P, et al. Classification of $\mathrm{R} 1$ resections for pancreatic cancer: the prognostic relevance of tumour involvement within $1 \mathrm{~mm}$ of a resection margin. Histopathology $2009 ; 55: 277-83$.

11. Esposito I, Kleeff J, Bergmann F, et al. Most pancreatic cancer resections are R1 resections. Ann Surg Oncol 2008;15:1651-60.

12. Verbeke CS. Resection margins and R1 rates in pancreatic cancer--are we there yet?Histopathology 2008;52:787-96.

13. Sauer R, Becker H, Hohenberger W, et al. Preoperative versus postoperative chemoradiotherapy for rectal cancer. N Engl J Med 2004;351:1731-40.

14. Colorectal Cancer Collaborative Group. Adjuvant

Cite this article as: Anderson JD, Wan W, Kaplan BJ, Myers J, Fields EC. Changing paradigm in pancreatic cancer: from adjuvant to neoadjuvant chemoradiation. J Gastrointest Oncol 2016;7(6):1004-1010. doi: 10.21037/jgo.2016.11.09 radiotherapy for rectal cancer: a systematic overview of 8,507 patients from 22 randomised trials. Lancet 2001;358:1291-304.

15. Minsky BD, Cohen AM, Kemeny N, et al. Combined modality therapy of rectal cancer: decreased acute toxicity with the preoperative approach. J Clin Oncol 1992;10:1218-24.

16. Sutton JM, Abbott DE. Neoadjuvant therapy for pancreas cancer: past lessons and future therapies. World J Gastroenterol 2014;20:15564-79.

17. Tzeng CW, Tran Cao HS, Lee JE, et al. Treatment sequencing for resectable pancreatic cancer: influence of early metastases and surgical complications on multimodality therapy completion and survival. J Gastrointest Surg 2014;18:16-24; discussion 24-5.

18. Gillen S, Schuster T, Meyer Zum Büschenfelde C, et al. Preoperative/neoadjuvant therapy in pancreatic cancer: a systematic review and meta-analysis of response and resection percentages. PLoS Med 2010;7:e1000267.

19. Stokes JB, Nolan NJ, Stelow EB, et al. Preoperative capecitabine and concurrent radiation for borderline resectable pancreatic cancer. Ann Surg Oncol 2011;18:619-27.

20. Katz MH, Pisters PW, Evans DB, et al. Borderline resectable pancreatic cancer: the importance of this emerging stage of disease. J Am Coll Surg 2008;206:83346; discussion 846-8.

21. Kim EJ, Ben-Josef E, Herman JM, et al. A multiinstitutional phase 2 study of neoadjuvant gemcitabine and oxaliplatin with radiation therapy in patients with pancreatic cancer. Cancer 2013;119:2692-700.

22. Stessin AM, Meyer JE, Sherr DL. Neoadjuvant radiation is associated with improved survival in patients with resectable pancreatic cancer: an analysis of data from the surveillance, epidemiology, and end results (SEER) registry. Int J Radiat Oncol Biol Phys 2008;72:1128-33.

23. Goodman KA, Hajj C. Role of radiation therapy in the management of pancreatic cancer. J Surg Oncol 2013;107:86-96. 